\title{
Immunostimulatory effects of the Pueraria lobata flower extract via MAPK signaling in RAW264.7 cells
}

\author{
Ki-tae Kim
}

Dept. of Internal Medicine, College of Korean Medicine, Semyung University

\begin{abstract}
Objectives: In this study, we investigated the effects of Pueraria lobata Ohwi flower extracts (PLFE) on macrophages and their underlying mechanism(s) of action. PLFE increased the production of NO and cytokines (IL- 6 and TNF- $a$ ) in a dose-dependent manner, indicating its immunostimulatory property. Furthermore, PLFE upregulated iNOS, COX-2, and mitogen-activated protein kinase (MAPK) signaling in RAW264.7 cells. Additionally, PLFE enhanced

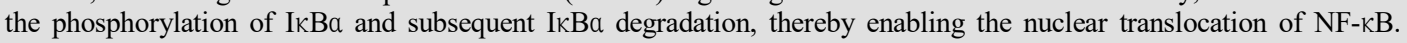
Taken together, these findings demonstrate that the immunostimulatory effects of PLFE are mediated by the nuclear translocation of the p65 subunit of NF-KB and subsequent secretion of cytokines (IL-6 and TNF- $\alpha$ ), upregulation of iNOS and COX-2, and stimulation of MAPK signaling (JNK, ERK, and p38). Thus, PLFE may be a potential immunostimulatory therapeutic.
\end{abstract}

Key Words : Pueraria lobata Ohwi flower, PLFE, immunosimulatory effect

\section{Introduction}

Immunity is an important and complex component of the defense mechanism of the body that comprises innate and adaptive immunity ${ }^{1)}$. Immune function refers to the improvement of biomarkers related to the enhancement of immune function and alleviation of irritable immune response. Immunity-enhancing functionality refers to a function that enhances the ability to protect the body by regulating immunity. The function of alleviating an overactive immune response refers to a function for suppressing an undesirably increased immune response, such as a reaction to an allergic reaction autoantigen or a modified autoantigen caused by a reaction to an external substance ${ }^{2)}$. Traditional oriental medicines have been widely used in alleviating immune-related diseases in the Asian population for a long time. There has been significant progress in research focusing on the enhancement of immunity using natural materials over the recent years. Ginseng and red ginseng have been used extensively for immune enhancements in Korea $^{3,4)}$. Chemotherapeutics inhibit the growth of cancer cells, but they can damage normal cells or cause side effects, such as immunosuppression ${ }^{5)}$. Moreover, the increase in particulate dust matter and other forms of environmental pollution results in specific

\footnotetext{
- Received : 9 November 2020

- Revised :

- Accepted : 17 November 2020

- Correspondence to : Ki-tae Kim

Dept. of Internal Medicine, College of Korean Medicine, Semyung University

Tel : +82-10-6649-6194, Fax : +82-43-645-1382, E-mail : onehorn@hanmail.net
} 
diseases. Therefore, the regulation of immune response plays an important role in the prevention and treatment of diseases. Macrophages are multifunctional cells that are crucial in innate and adaptive immunity ${ }^{6}$. During pathogen invasion, the adaptive immune system cooperates with innate immunity to eliminate pathogenic microorganisms ${ }^{7)}$.

Lipopolysaccharide (LPS) activates macrophages. LPS binds to toll-like receptor 4 (TLR4) that primarily directs the immune response by activating the macrophages and secreting cytokines ${ }^{8)}$. Activated macrophages secrete a variety of cytokines and chemokines, such as interferon- $\gamma$, interleukin (IL)-1 $\beta$, IL-6, IL-12, tumor necrosis factor (TNF)- $a$, nitric oxide (NO), and prostaglandin $\mathrm{E}_{2}{ }^{9)}$. Thus, it is imperative to understand macrophage-mediated immunity.

Pueraria lobata belongs to the Leguminosae family. The roots and flowers of $P$. lobata are widely used in traditional medicine ${ }^{10,11)}$ primarily for cardiovascular diseases, hangover, inappetence, and vomiting ${ }^{11,12)}$. Previous studies have shown that the active compounds of $P$. lobata extract comprise isoflavones, including kakkalide, kakkalidone, puerarin, irisolidone, 6" -glycitin, tectoridin, 6"-O-xylosyl-tectoridin, and genistin $^{13,14,15)}$.

Most studies on the extract of $P$. lobata have focused on its effects as an antioxidant, anti-inflammatory agent, and hangover remedy 10,11,16). However, the immune activity of the extract remains to be studied. Therefore, we wanted to understand the immune effects of the extract of $P$. lobata flowers. In this study, we report the immuopotentiating effects of the $P$. lobata flower extract (PLFE) on RAW264.7 cells based on the production of NO and cytokines and NF-кB/MAPK signaling.

\section{Materials and Methods}

\section{Materials}

Lipopolysaccharide (LPS), 3-(4,5-dimethylthiazol -2-yl)-2,5-diphenyltetrazolium bromide (MTT) were purchased from Sigma Chemical Co. (St. Louis, MO, USA). P. lobata flowers were obtained from Hanbang Herbal Drug Co. (Jecheon, Korea). Bradford reagent was procured from BioRad (Hercules, CA, USA). Antibodies against iNOS and COX-2 were purchased from Santa Cruz Biotechnology, Inc. (Santa Cruz, CA, USA);

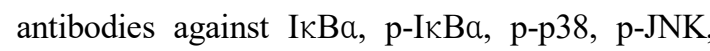
and p-ERK were obtained from Cell Signaling Technology (Beverly, MA, USA). All other reagents were of the highest grade commercially available.

\section{Preparation of PLFE}

PLFE was prepared as described. Briefly, dried P. lobata flowers (100 g) were extracted with distilled water. The water extract was obtained by refluxing the sample for $90 \mathrm{~min}$ at room temperature followed by suction filtering through filter paper (Whatman No. 41). The filtered extracts were evaporated under reduced pressure using a rotary vacuum evaporator (EYELA, Tokyo, Japan) at $50^{\circ} \mathrm{C}$. After evaporation, the water and ethanol extracts were lyophilized using a freeze dryer (Samwon Industry, Seoul, Korea).

\section{Cell culture}

Murine macrophage RAW264.7 cells were purchased from the Korean Cell Line Bank (Seoul, Korea) and maintained in high glucose Dulbecco's modified Eagle's medium supplemented 
with $10 \%$ heat-inactivated fetal bovine serum, 100 $\mathrm{U} / \mathrm{mL}$ penicillin, and $100 \mu \mathrm{g} / \mathrm{mL}$ streptomycin at $37^{\circ} \mathrm{C}$ in a humidified incubator $\left(5 \% \mathrm{CO}_{2}\right.$ and $95 \%$ air).

\section{Cell viability}

The cytotoxic effects of the extracts on RAW264.7 cells were evaluated using the MTT assay. Briefly, RAW264.7 cells were seeded in 96-well plates at a density of $2 \times 10^{4}$ cells/well. Cells were treated with $0,25,50$, or $100 \mu \mathrm{g} / \mathrm{mL}$ of PLFE or LPS (100 ng/mL) for $24 \mathrm{~h}$. The cells were then incubated with MTT solution for $2 \mathrm{~h}$. Subsequently, the supernatant was removed and resulting formazan crystals were dissolved in dimethyl sulfoxide before absorbance at $540 \mathrm{~nm}$ was measured using a microplate reader (SpectraMax M2/M2e, Molecular Devices, Sunnyvale, CA, USA).

\section{Measurement of NO generation}

NO levels in the cell culture medium were analyzed by determining the accumulation of nitrite in the cell culture medium using the Griess reagent and sodium nitrite as the standard. RAW264.7 cells were treated with PLFE for 18 $\mathrm{h}$ before the culture medium $(100 \mu \mathrm{L})$ was transferred into each well of a 96-well plate. An equal volume $(100 \mu \mathrm{L})$ of Griess reagent was added to each well and incubated for $10 \mathrm{~min}$ at room temperature. Finally, the absorbance at 550 nm was measured using a microplate reader (SpectraMax M2/M2e, Molecular Devices, Sunnyvale, CA, USA).

\section{Inflammatory cytokine production}

RAW264.7 cells $\left(1 \times 10^{5}\right.$ cells/well $)$ were seeded into 24-well plates and incubated overnight for cell adherence. After treatment with PLFE or LPS (positive control) for $18 \mathrm{~h}$, TNF- $\mathrm{a}$ and IL-6 levels in the cell supernatants was assayed using enzyme-linked immunosorbent assay (R\&D Systems, Minneapolis, MN, USA) according to the manufacturer's instructions.

\section{Western blotting}

Treated cells were harvested, lysed, and subjected to protein quantification using the Bradford assay. Samples containing $15-20 \mu \mathrm{g}$ of proteins were subjected to sodium dodecyl sulfate -polyacrylamide gel electrophoresis (BioRad Laboratories, Hercules, CA, USA) and separated proteins were transferred to polyvinylidene fluoride membranes (GE Healthcare, Buckinghamshire, UK). After blocking with 5\% skim milk in Tris-buffered saline supplemented with Tween-20 for $1 \mathrm{~h}$ at room temperature, the membranes were incubated overnight with specific primary antibodies at $4^{\circ} \mathrm{C}$. After washing thrice with the Tris-buffered saline with Tween-20, the membranes were incubated with species-specific horseradish peroxidase-conjugated secondary antibodies for 1 $\mathrm{h}$ at room temperature. Protein bands were developed using enhanced chemiluminescence reagents and the immunoblots were visualized using the Davinch-Chemi ${ }^{\mathrm{TM}}$ imaging system (Young Wha Scientific Co. Ltd, Seoul, Korea). Densitometry for the target protein levels were normalized to $\beta$-actin.

\section{Statistical analysis}

The results are expressed as the mean \pm standard deviation (S.D.) values. Statistical analysis was performed by analysis of variance (ANOVA), followed by Dunnett's test to determine the significance of differences using GraphPad Prism 

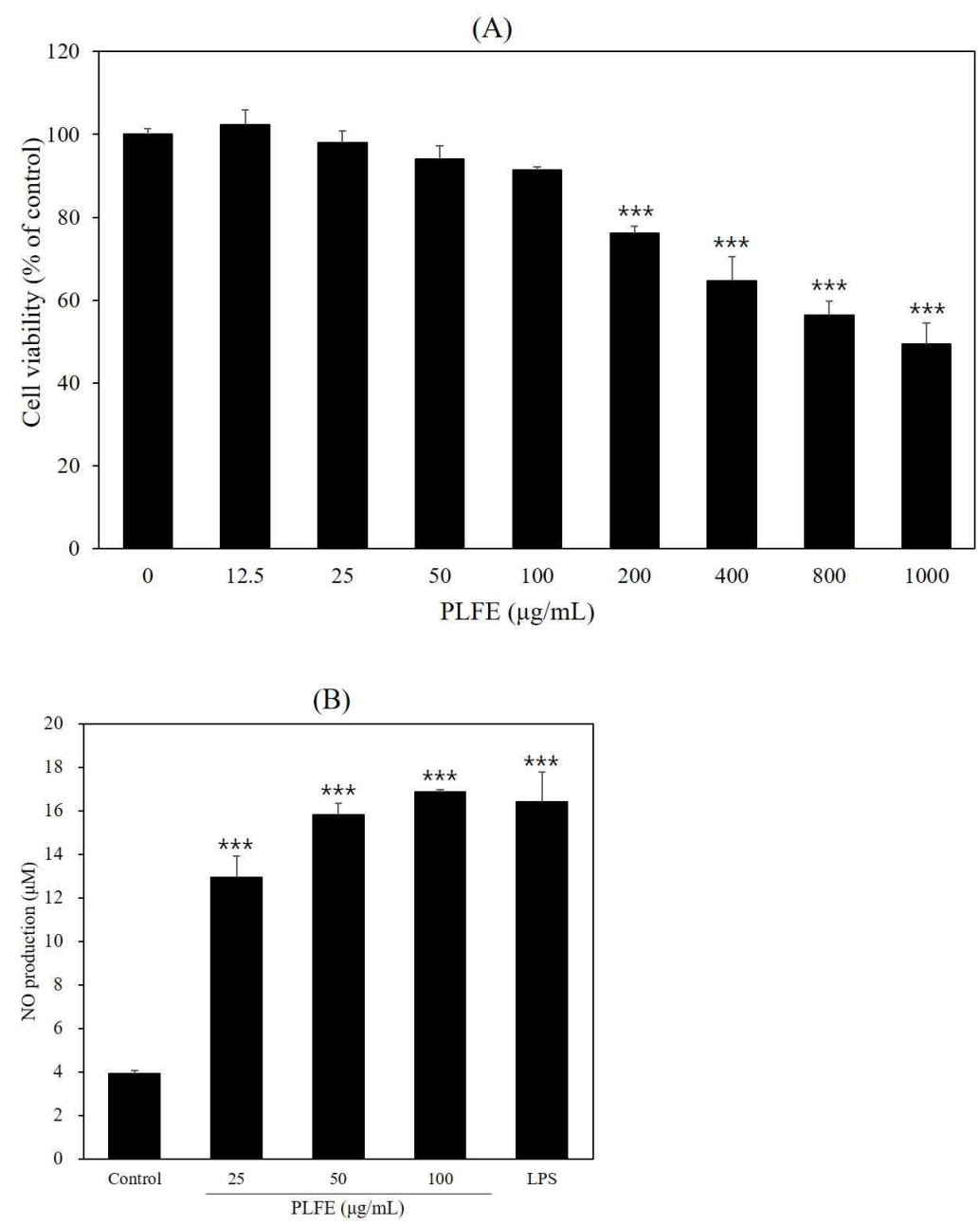

Fig. 1. PLFE was not toxic under $100 \mu \mathrm{g} / \mathrm{mL}$ (A) and PLFE induced the production of NO RAW264.7 macrophage cells (B). All values are expressed as the means \pm S.D. ${ }^{* * *} p<0.001$ vs. control.

5.0, $p<0.05$ was considered statistically significant.

\section{Results}

1. Effect of PLFE on cell viability and NO production in RAW264.7 cells

To determine the effect of PLFE on the cell viability, cells were treated with various concentration of PLFE $(0-1,000 \mu \mathrm{g} / \mathrm{mL})$. PLFE showed over $90 \%$ of cell viability below $100 \mu$ $\mathrm{g} / \mathrm{mL}$ concentration and didn't showed toxicity (Fig. 1A). Therefore, we performed the following experiments with the experimental concentration of $100 \mu \mathrm{g} / \mathrm{mL}$ as the highest concentration. To assess the enhanced-immune effect of PLFE, we examined whether PLFE affected the NO production in RAW264.7 cells. As shown Fig. 1B, 

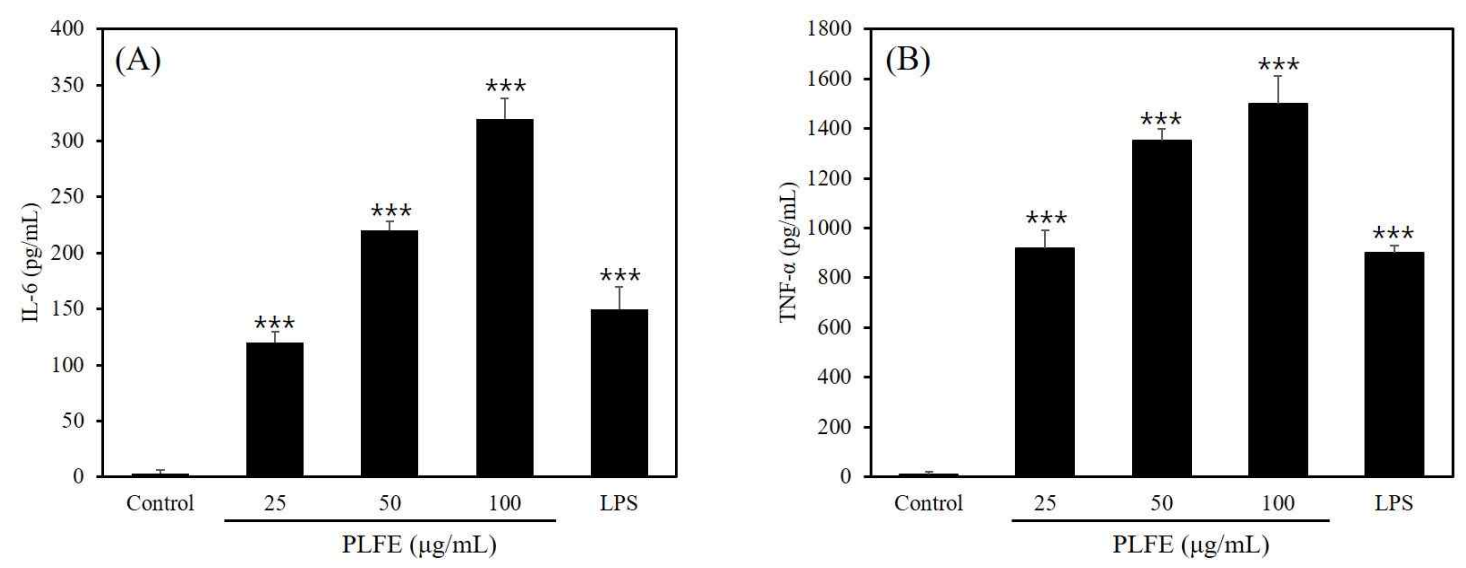

Fig. 2. PLFE increased the production of cytokines (IL-6(A), TNF $-a(B)$ ). The release of cytokines was determined by ELISA assay. All values are expressed as the means \pm S.D. ${ }^{* * * *} p<0.001$ vs. control.

PLFE increased the NO production in dose-dependent manner. Compare with the control group, NO production was markedly increased $(p<0.001)$ the treatment of PLFE as much as LPS group. Thus, only PLFW was subjected to further analysis to explore its immuno-stimulating effect and mechanisms.

\section{Effect of PLFE on the expressions of $\mathrm{TNF}-a$ and IL-6}

Cytokines are important mediators involved in modulating immune response and inflammatory reactions, especially during infection and trauma 17). The cytokines, including TNF- $\alpha$ and IL-6, are potent immunomodulators in activated macrophages. Thus, we determined if PLFE affected the expression of cytokines in RAW264.7 cells. As shown in Fig. 2, compared with the control group, IL-6 and TNF-a levels increased in the PLFE-treated group $(p<0.001)$. The amount of cytokines secreted from PLFE -treated cells was higher than that from LPS-treated cells (positive control). These results suggest that PLFE promotes immunostimulation in RAW264.7 cells.

\section{Effect of PLFE on iNOS and COX-2 expression}

To further demonstrate the immune stimulatory effect of PLFE, we determined the protein levels of iNOS and COX-2 using western blotting. As shown in Fig. 3, the protein levels of iNOS and COX-2 increased in PLFE-treated cells in a dose-dependent manner. Cells treated with various concentrations of PLFE (25, 50, and 100 $\mu \mathrm{g} / \mathrm{mL}$ ) for $18 \mathrm{~h}$ significantly increased iNOS and COX-2 protein levels compared to those in the control. We used LPS as a positive control to show the strong expression of iNOS and COX-2 compared to the control sample. In addition, PLFE induced the expression of iNOS and COX-2 stronger than that observed in LPS-treated cells. Thus, PLFE may upregulate pro-inflammatory mediators, such as iNOS and COX-2.

\section{Effect of PLFE on IkBa degradation}


(A)

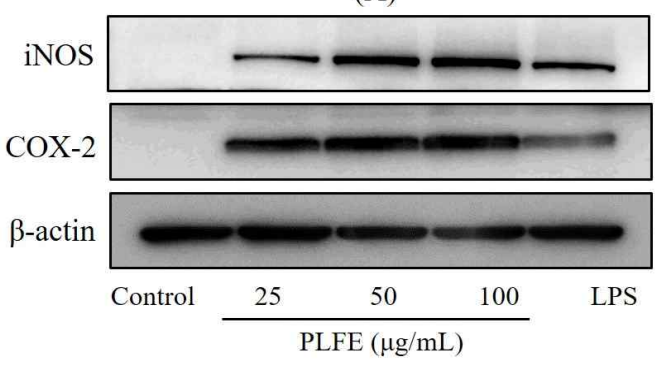

(B)

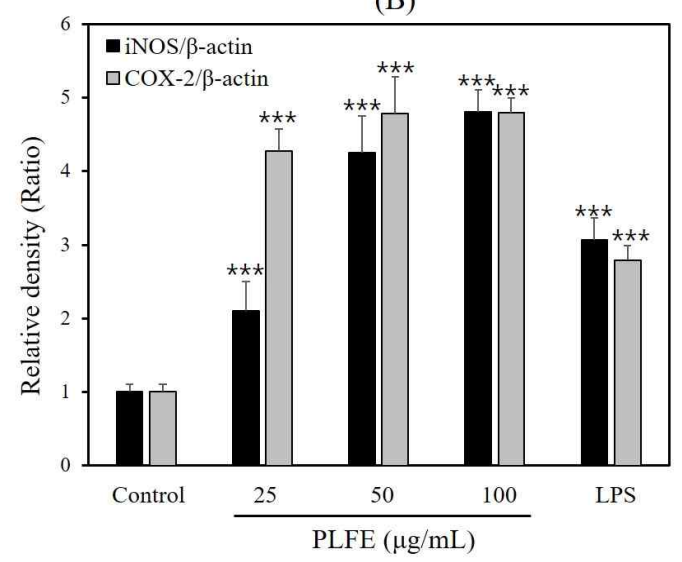

Fig. 3. PLFE induced iNOS and COX-2 protein expression. Cells were treated with PLFE or LPS $(100 \mathrm{ng} / \mathrm{mL})$ for $18 \mathrm{~h}$ and protein expression levels were determined by Western blot analysis (A) and quantified using Image J (B). All values are expressed as the means \pm S.D. ${ }^{* *} p$ $<0.05$ and ${ }^{* * *} p<0.001$ vs. control.

I $\mathrm{B} a$ degradation is crucial in the activation of the transcription factor NF- $\kappa \mathrm{B}$. Thus, we investigated

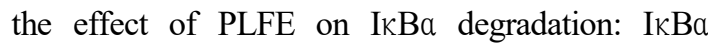
protein levels decreased in PLFE or LPS-treated cells (Fig. 4A, 4B). In addition, PLFE increased the phosphorylation of $\mathrm{I} \kappa \mathrm{B} a$. I $\mathrm{K} \mathrm{B} a$ constitutes a family of cellular proteins that inhibit $N F-\kappa B$ activity by masking the nuclear localization signal of NF- $\mathrm{B}$ proteins and sequestering their inactive forms in the cytoplasm ${ }^{18)}$. External stimuli that activate macrophages enhance I $\kappa \mathrm{B} a$ phosphorylation,
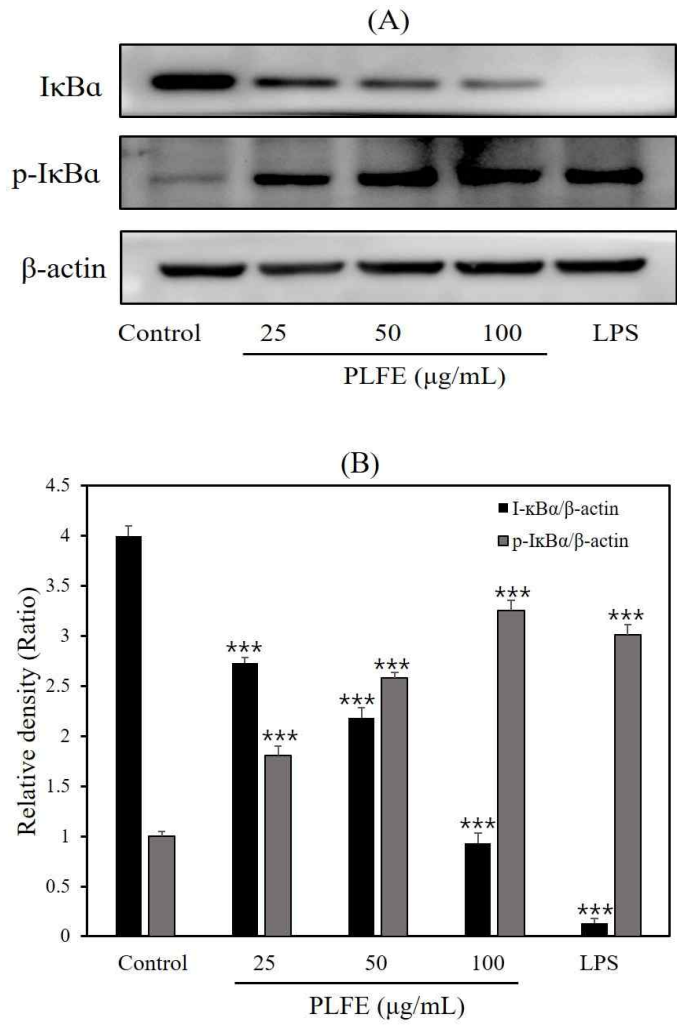

Fig. 4. PLFE degraded I $\mathrm{I} B a$ expression and increased phosphorylation levels of $\mathrm{I} \mathrm{Ba}$ expression. Cells were treated with PLFE or LPS (100 ng/mL) for $15 \mathrm{~min}$. Total cellular proteins were prepared and subjected to Western blot analysis for determination of $\mathrm{I} \kappa \mathrm{Ba}$ and $\mathrm{p}$-IкBa protein levels (A) and quantified using ImageJ (B). All values are expressed as the means \pm S.D. ${ }^{* *} p$ $<0.05$ and ${ }^{* * *} p<0.001$ vs. control.

leading to its degradation and simultaneous activation and nuclear translocation of NF- $\mathrm{kB}$ to bind to its cognate DNA-binding site in the regulatory region of multiple genes ${ }^{19)}$. Therefore, our results suggested that PLFE activates NF- $\kappa B$, thereby enabling the target gene transcription in macrophages.

\section{Effect of PLFE on MAPK signaling}

To investigate whether PLFE affects immunity 
(A)

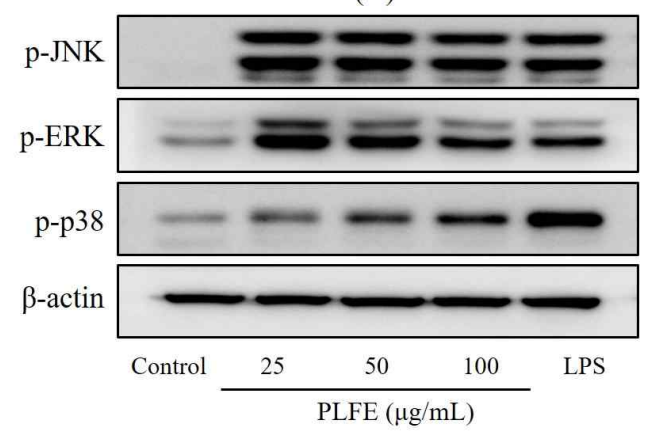

(C)

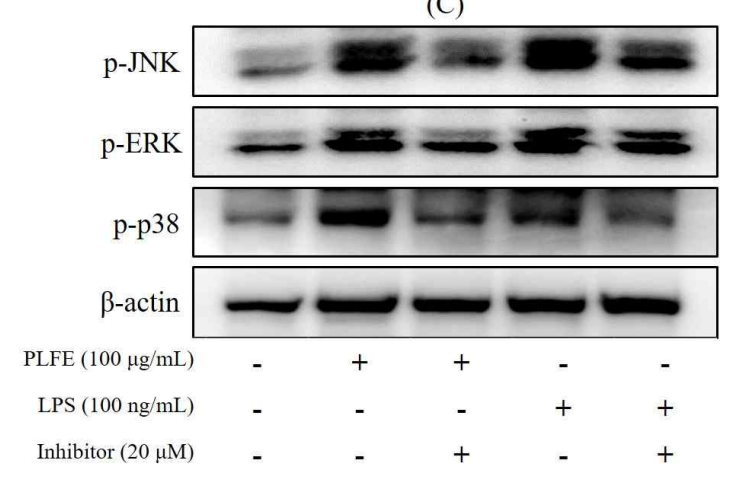

(B)

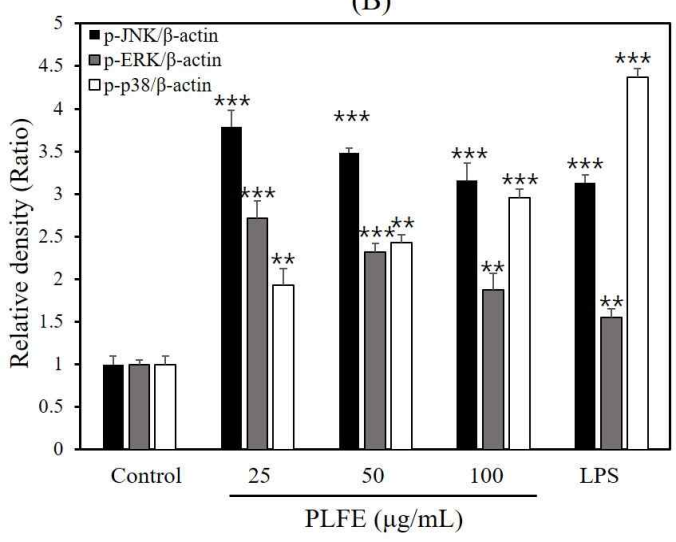

(D)

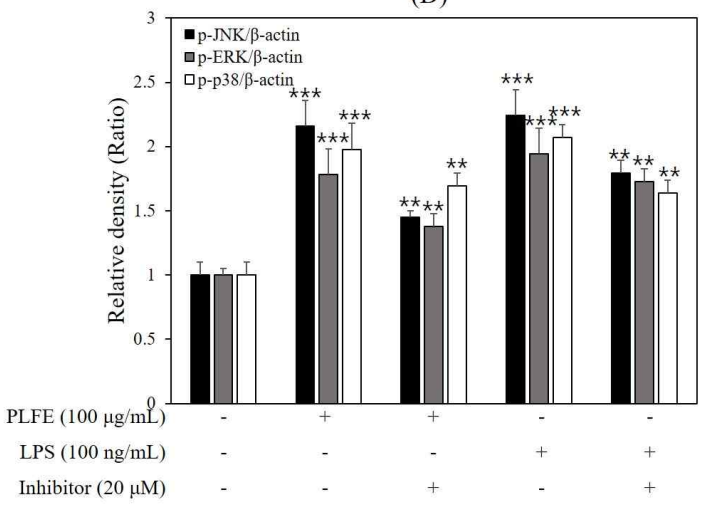

Fig. 5. PLFE increased on MAPK signaling pathway. Cells were treated with PLFE or LPS (100 ng/mL) for 30 min and MAPK protein expression levels were determined by Western blot analysis with specific antibodies (A) and quantified using ImageJ (B). Cells were treated with PLFE or LPS $(100 \mathrm{ng} / \mathrm{mL})$ in the presence of MAPK inhibitors for $30 \mathrm{~min}$ and MAPK protein expression levels were determined (C) and quantified using ImageJ (D). Cells were treated with $20 \mu \mathrm{M}$ of the MAPK inhibitor SB203580 (p38 inhibitor), SP600125 (JNK inhibitor), and PD098059 (ERK inhibitor). All values are expressed as the means \pm S.D. ${ }^{* *} p<0.05$ and ${ }^{* * * *} p<0.001$ vs. control.

via MAPK signaling, we evaluated the protein levels of the MAPK family of proteins, including p38, JNK, and ERK1/2 using western blot analysis. As shown in Fig 5A, 5B, 30 min of PLFE and LPS increased the phosphorylation of MAPK isoforms, p38, JNK, and ERK1/2. In addition, MAPK expression was inhibited in cells treated with PLFE and MAPK-specific inhibitor (Fig. 5C, 5D). Taken together, PLFE stimulated MAPK signaling, thereby upregulating iNOS and COX-2 by activating NF- $\mathrm{kB}$.

\section{Discussion}

Macrophages comprise an integral component of the first line of defense in protecting the host from infection ${ }^{20)}$ by eliciting immune responses ${ }^{21)}$. Macrophages are one of the important innate 
immune cells that make up our body, and are distributed in various forms in all tissues, and under normal conditions, they play a role in protecting the body through the predation of invading foreign pathogens and toxic substances.

Since abnormalities in the function of macrophages can cause various diseases, research on macrophages is being actively conducted. M1 macrophages are a type of macrophage with typical functions, and M2 macrophages are alternative activated macrophages with a wide range of physiological pathological effects. M2 macrophages have different functions than M1 macrophages, are important for cell homeostasis and inflammatory reactions, and have specific effects on tissue repair or metabolic processes. Research on M2 macrophages has been actively conducted in recent years, and various subtypes have been discovered and reported. It is polarized by specific substances or various kinds of reactions and is subdivided into new types of M2 macrophages such as M2a, M2b, M2c, and $\mathrm{M} 2 \mathrm{~d}^{22)}$. In addition, it has been reported that various types of macrophages have an important influence on not only immune function but also metabolic regulation ${ }^{23)}$. Contrary to the common knowledge that immune cells will suppress cancer, studies have also been reported on macrophages that promote tumor development, such as Tumor-associated macrophage (TAM) ${ }^{24)}$. NO is a signaling molecule that is secreted by macrophages, neurons, and endothelial cells and function in protecting or being toxic to cells under specific conditions ${ }^{25,26)}$. This study showed that PLFE increased the production of NO by upregulating iNOS in macrophages. We concluded that PLFE regulated the synthesis of pro-inflammatory cytokines in RAW264.7 cells.
Cytokines are secretory proteins from immune and interstitial cells involved in intercellular communication and exchange of biomolecules. Cytokine function is classified based on their pro-inflammatory or anti-inflammatory effects and associated signal transduction is involved in the regulation of immune response ${ }^{27,28)}$. IL-10 is an anti-inflammatory cytokine, while IL-6 and TNF-a are pro-inflammatory cytokines that comprise a key area of research in immunology 29).

IL-6 is a soluble protein with pleiotropic effects on inflammation, immune response, and hematopoiesis $^{30)}$ and is secreted from numerous cells, including $\mathrm{T}$ cells, $\mathrm{B}$ cells, mononuclear phagocytes, fibroblasts, hepatocytes, endothelial cells, keratinocytes, and bone marrow cells ${ }^{31)}$. IL-6 stimulates $\mathrm{T}$ cell proliferation and, together with IL-4, participates in the generation of the Th2 immune response ${ }^{32,33)}$. IL-6 also participates in triggering humoral immunity by stimulating $\mathrm{B}$ cell differentiation and antibody secretion ${ }^{34)}$.

TNF- $\alpha$ is a central regulator of inflammation produced by neutrophils, lymphocytes, and natural killer, endothelial, and mast cells ${ }^{35)}$. The soluble active form of TNF-a is cleaved from its membrane-bound form using the TNF- $a$ converting enzyme $^{36)}$. TNF- $\alpha$ and TNF- $\beta$ induce antitumor immunity via direct cytotoxic effects on cancerous cells ${ }^{37)}$. In this study, we investigated the immune-enhancing activity of PLFE based on cytokine production in murine macrophage cells. We have demonstrated that PLFE enhanced the synthesis of IL-6 and TNF-a in a dose-dependent manner. PLFE may exert its immunostimulatory effect by increasing the secretion of NO and cytokines. MAPK (p38, JNK, and ERK) signaling is upstream of IL-1 
IL-6, and TNF-a synthesis ${ }^{38,39)}$. PLFE-treated RAW264.7 cells showed an upregulation of MAPK pathway components. In order to confirm the immunostimulatory effect, IL-6 and TNF-a were measured, and IL-10, a deduction inhibitory cytokine, was not measured, but it will have to be confirmed in further experiments.

$\mathrm{NF}-\kappa \mathrm{B}$ is a transcription factor for immune response-related gene expression and plays an important role in the immune function of monocytes and macrophages ${ }^{40)}$. I $\kappa \mathrm{Ba}$ inhibits $\mathrm{NF}-\kappa \mathrm{B}$ by masking its nuclear localization signal and retaining an inactive form of $\mathrm{NF}-\mathrm{\kappa B}$ in the cytoplasm $^{18)}$. This study showed that PLFE decreased $\mathrm{I} \kappa \mathrm{B} \alpha$ and increased the phosphorylation of I $\kappa \mathrm{B} a$. Therefore, PLFE activated NF- $\kappa \mathrm{B}$ by degrading I $\mathrm{K} \mathrm{B} \alpha$. In order to find out this mechanism more clearly, the expression of NF-kB and $\mathrm{p}-\mathrm{NF}-\mathrm{kB}$ should be confirmed.

According to the health functional food functional evaluation guide proposed by the Ministry of Food and Drug Safety, the function related to the improvement of immune function is evaluated in terms of two aspects: enhancing immune function and helping to alleviate hypersensitivity immune response ${ }^{2)}$. This study was designed to improve immune function, and analysis of the components of PLFE and animal experiments should be conducted in the future.

\section{Conclusions}

We analyzed the effects of PLFE on immune regulation in RAW264.7 cells. PLFE enhanced the immune activity of the murine macrophage RAW264.7 cells by activating NF- $\mathrm{B}$ /MAPK signaling, thereby increasing the secretion of NO and cytokines (IL-6 and TNF- $\alpha$ ). In summary, we have identified the main components involved in the immunostimulatory and immune enhancement effects of PLFE using an in vivo model.

\section{Acknowledgement}

This paper was supported by the Semyung University Research Grant 2020.

\section{References}

1. Haque, M.A.; Jantan, I.; Bukhari, S.N.A. Tinospora species: An overview of their modulating effects on the immune system. Journal of Ethnopharmacology 2017, 207, 67-85.

2. Sunhee Park, Changsook Yang, Heesun Kim, Yunju Choi, Miran Jang, Yongmoo Kim, Minsik Kim, Yoonjung Kang, Taeseok Kang, Hwajeong Lee, Gyungneo Ban, Ilwon Seo, Youngju Lee, Jinhee Lee, Jaemyung Oh. Health Functional Food Functional Evaluation Guide-'Can help improve immune function' section. Ministry of Food and Drug Safety 2014, 15-16.

3. Reyes, A.W.B.; Hop, H.T.; Arayan, L.T.; Huy, T.X.N.; Park, S.J.; Kim, K.D. The host immune enhancing agent Korean red ginseng oil successfully attenuates Brucella abortus infection in a murine model. Journal of Ethnopharmacology 2017, 198, 5-14.

4. Hong, Y.J.; Kim, N.; Lee, K.; Sonn, C.H.; Lee, J.E.; Kim, S.T.; Baeg, I.H.; Lee, K.M. Korean red ginseng (Panax ginseng) ameliorates type 1 diabetes and restores immune cell compartments. Journal of Ethnopharmacology 2012, 144, 225-233. 
5. Hong, S.H.; Ku, J.M.; Kim, H.I.; Ahn, C.W.; Park, S.H.; Seo, H.S.; Shin, Y.C.; Ko, S.G. The immune-enhancing activity of Cervus nippon mantchuricus extract (NGE) in RAW264.7 macrophage cells and immunosuppressed mice. Food Research International 2017, 99, 623-629.

6. Wu, F.; Zhou, C.; Zhou, D.; Ou, S.; Liu, Z.; Huang, H. Immune-enhancing activities of chondroitin sulfate in murine macrophage RAW 264.7 cells. Carbohydrate Polymers 2018, 198, 611-619.

7. Tang, C.; Sun, J.; Liu, J.; Jin, C.; Wu, X.; Zhang, X.; Chen, H.; Gou, Y.; Kan, J.; Qian, C.; Zhang, N. Immune-enhancing effects of polysaccharides from purple sweet potato. International Journal of Biological Macromolecules 2019, 123, 923-930.

8. Han, S.B.; Yoon, Y.D.; Ahn, H.J.; Lee, H.S.; Lee, C.W.; Yoon, W.K.; Park, S.K; Kim, H.M. Toll-like receptor-mediated activation of $\mathrm{B}$ cells and macrophages by polysaccharide isolated from cell culture of Acanthopanax senticosus. International Immunopharmacology 2003, 3, 1301-1312.

9. Hong, S.H.; Ku, J.M.; Kim, H.I.; Ahn, C.W.; Park, S.H.; Seo, H.S.; Shin, Y.C.; Ko, S.G. The immune-enhancing activity of Cervus nippon mantchuricus extract (NGE) in RAW264.7 macrophage cells and immunosuppressed mice. Food Research International 2017, 99, 623-629.

10. McGregor, N.R. Pueraria lobata (Kudzu root) hangover remedies and acetaldehyde -associated neoplasm risk. Alcohol 2007, 41, 469-478.

11. Shi, S.; Ma, Y.; Zhang, Y.; Liu, L.; Liu, Q.; Peng, M.; Xiong, X. Systematic separation and purification of 18 antioxidants from Pueraria lobata flower using HSCCC target-guided by DPPH-HPLC experiment. Separation and Purification Technology 2012, 89, 225-233.

12. Bebrevska, L.; Foubert, K.; Hermans, N.; Chatterjee, S.; Van Marck, E.; De Meyer, G.; Vlietinck, A.; Pieters, L.; Apers, S. In vivo antioxidative activity of a quantified Pueraria lobata root extract. Journal of Ethnopharmacology 2010, 127, 112-117.

13. Yao, M.; Liao, Y.; Li, G.Q.; Law, F.C.; Tang, Y. Quantitative analysis of two isoflavones in Pueraria lobata flowers from eleven Chinese provinces using high performance liquid chromatography. Chinese Medicine 2010, 5, 14. doi: 10.1186/1749-8546-5-14.

14. Pei, X.P.; Pei, M.R.; Ding, H.Q. Chemical constituents from Pueraria lobata. Shanxi Daxue Xuebao 2010, 33, 423-424.

15. Kinjo, J.; Takeshita, T.; Abe, Y.; Terada, N.; Yamashita, H.; Yamasaki, M.; Takeuchi, K.; Murakami, K.; Tomimatsu, T.; Nohara, T. Studies on the Constituents of Pueraria lobata. IV. : Chemical Constituents in the Flowers and the Leaves. Chemical and Pharmaceutical Bulletin 1988, 36, 1174-1179.

16. Jin, S.E.; Son, Y.K.; Min, B.S.; Jung, H.A.; Choi, J.S. Anti-inflammatory and antioxidant activities of constituents isolated from Pueraria lobata roots. Archives of Pharmacal Research 2012, 35(5), 823-837.

17. Wang, J.; Nie, S.; Cui, S.W.; Wang, Z.; Phillips, A.O.; Phillips, G.O.; Li, Y.; Xie, M. Structural characterization and immunostimulatory activity of a glucan from natural Cordyceps sinensis. Food Hydrocolloids 2017, 67, 139-147. 
18. Jacobs, M.D.; Harrison, S.C. Structure of an

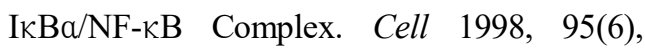
749-758.

19. Suh, S.J.; Chung, T.W.; Son, M.J.; Kim, S.H.; Moon, T.C.; Son, K.H.; Kim, H.P.; Chang, H.W.; Kim, C.H. The naturally occurring biflavonoid, ochnaflavone, inhibits LPS-induced iNOS expression, which is mediated by ERK1/2 via NF- $\kappa$ B regulation, in RAW264.7 cells. Archives of Biochemistry and Biophysics 2006, 447, 136-146.

20. Shu, Y.; Liu, X.B.; Ma, X.H.; Gao, J.; He, W.; Cao, X.Y.; Chen, J. Immune response mechanism of mouse monocytes/macrophages treated with $\mathrm{k}$-carrageenan polysaccharide. Environmental Toxicology and Pharmacology 2017, 53, 191-198.

21. Li, Y.; Meng, T.; Hao, N.; Tao, H.; Zou, S.; Li, M.; Ming, P.; Ding, H.; Dong, J.; Feng, $\mathrm{S}$;; Li, J.; Wang, X.; Wu, J. Immune regulation mechanism of Astragaloside IV on RAW264.7 cells through activating the NF- $\mathrm{B} / \mathrm{MAPK}$ signaling pathway. International Immunopharmacology 2017, 49, 38-49.

22. In-mu Lee.Cancer and immunity-Development of anticancer immunotherapy using macrophages. Sejong science \& techologly newsletter October 2016 Vol.11

23. Camell C. D., Sander J., Spadaro O., Lee A., Nguyen K. Y., Wing A., Goldberg E. L., Youm Y., Brown C. W., Elsworth J., Rodeheffer M. S., Schultze J. L. and Dixit V. D. Inflammasome-driven catecholamine catabolism in macrophages blunts lipolysis during ageing. Nature 2017;550(7674): 119-123.

24. Eun Jo. Tumor-Associated Macrophages: From Mechanisms to Therapy. Immunity, 2014;41(1): 49-61.
25. Gamper, N.; Ooi, L. Redox and nitric oxide-mediated regulation of sensory neuron ion channel function. Antioxidants \& Redox Signaling 2015, 22 (6), 486-504.

26. Steiner, N.; Balez, R.; Karunaweera, N.; Lind, J.M.; Münch, G.; Ooi, L. Neuroprotection of Neuro2a cells and the cytokine suppressive and anti-inflammatory mode of action of resveratrol in activated RAW264.7 macrophages and C8-B4 microglia. Neurochemistry International 2016, 95, 46-54.

27. Shachar, I.; Karin, N. The dual roles of inflammatory cytokines and chemokines in the regulation of autoimmune diseases and their clinical implications. Journal of Leukocyte Biology 2013, 93, 51-61.

28. Shuai, K.; Liu, B. Regulation of JAK-STAT signalling in the immune system. Nature Reviews Immunology 2003, 3, 900-911.

29. Yu, Z.M.; Huang, X.H.; Yan, C.Q.; Gao, J.; Liang, Z.S. Effect of Fuzheng Jiedu granule on immunological function and level of immune-related cytokines in immune -suppressed mice. Journal of Integrative Agriculture 2016, 15(3), 650-657.

30. Tanaka, T.; Narazaki, M.; Kishimoto, T. IL-6 in Inflammation, Immunity, and Disease. Cold Spring Harbor Perspectives in Biology 2014, 6, a016295

31. Jücker, M.; Abts, H.; Li, W.; Schindler, R.; Merz, H.; Günther, A.; von Kalle, C.; Schaadt, M.; Diamantstein, T.; Feller, A.C.; Krueger, G.R.F.; Diehl. V.; Blankenstein, T.; Tesch, H. Expression of Interleukin-6 and Interleukin-6 Receptor in Hodgkin's Disease. Blood 1991, 77, 2413-2418.

32. Newman, R.E.; Yoo, D.; LeRoux, M.A.; Danilkovitch-Miagkova, A. Treatment of 
Inflammatory Diseases with Mesenchymal Stem Cells. Inflammation \& Allergy-Drug Targets 2009, 8, 110-123.

33. Xing, Z.; Gauldie, J.; Cox, G.; Baumann, H.; Jordana, M.; Lei, X.F.; Achong, M.K. IL-6 is an antiinflammatory cytokine required for controlling local or systemic acute inflammatory responses. Journal of Clinical Investigation 1998, 101, 311-320.

34. Kimura, A.; Kishimoto, T. IL-6: regulator of Treg/Th17 balance. European Journal of Immunology 2010, 40, 1830-1835.

35. Esposito, E.; Cuzzocrea, S. TNF-alpha as a therapeutic target in inflammatory diseases, ischemia- reperfusion injury and trauma. Current Medicinal Chemistry 2009, 16(24), 3152-3167.

36. Perez, C.; Albert, I.; DeFay, K.; Zachariades, N.; Gooding, L.; Kriegler, M., A nonsecretable cell surface mutant of tumor necrosis factor (TNF) kills by cell-to-cell contact. Cell 1990, 63, 251-258.

37. Commins, S.P.; Borish, L.; Steinke, J.W. Immunologic messenger molecules: Cytokines, interferons, and chemokines. Journal of Allergy and Clinical Immunology 2010, 125(2), S53-S72.

38. Cho, J.W.; Lee, K.S.; Kim, C.W. Curcumin attenuates the expression of IL-1 $\beta$, IL-6, and TNF- $\alpha$ as well as cyclin E in TNF- $\alpha$-treated $\mathrm{HaCaT}$ cells; NF- $\mathrm{BB}$ and MAPKs as potential upstream targets. International journal of molecular medicine 2007, 19, 469-474.

39. Fisher, W.G.; Yang, P.C.; Medikonduri, R.K.; Jafri, M.S. NFAT and NFkB activation in $\mathrm{T}$ lymphocytes: a model of differential activation of gene expression. Annals of Biomedical Engineering 2006, 34, 1712-1728.

40. Guha, M.; Mackman, N. LPS induction of gene expression in human monocytes. Cellular Signalling 2001, 13, 85-94.

\section{ORCID}

Ki-tae Kim https://orcid.org/0000-0003-4892-3472 